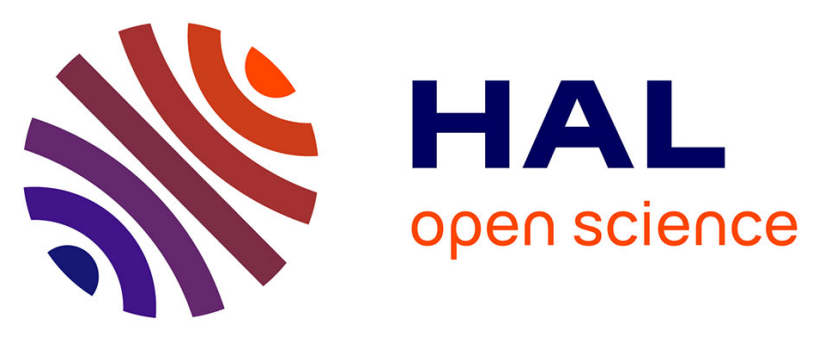

\title{
The Influence of Indenter Tip Imperfection and Deformability on Analysing Instrumented Indentation Tests at Shallow Depths of Penetration on Stiff and Hard Materials
}

Vincent Keryvin, Ludovic Charleux, Cédric Bernard, Mariette Nivard

\section{To cite this version:}

Vincent Keryvin, Ludovic Charleux, Cédric Bernard, Mariette Nivard. The Influence of Indenter Tip Imperfection and Deformability on Analysing Instrumented Indentation Tests at Shallow Depths of Penetration on Stiff and Hard Materials. Experimental Mechanics, 2017, 57 (7), pp.1107-1113. 10.1007/s11340-017-0267-1 . hal-01520783

\section{HAL Id: hal-01520783 \\ https://hal.science/hal-01520783}

Submitted on 18 Jul 2017

HAL is a multi-disciplinary open access archive for the deposit and dissemination of scientific research documents, whether they are published or not. The documents may come from teaching and research institutions in France or abroad, or from public or private research centers.
L'archive ouverte pluridisciplinaire HAL, est destinée au dépôt et à la diffusion de documents scientifiques de niveau recherche, publiés ou non, émanant des établissements d'enseignement et de recherche français ou étrangers, des laboratoires publics ou privés. 


\title{
The Influence of Indenter Tip Imperfection and Deformability on Analysing Instrumented Indentation Tests at Shallow Depths of Penetration on Stiff and Hard Materials
}

\author{
V. Keryvin - L. Charleux - C. Bernard - M. Nivard
}

Abstract We report on the difficulties encountered when extracting plastic parameters from constitutive equations by instrumented indentation testing on hard and stiff materials at shallow depths of penetration. As a general guidance, we refer to materials exhibiting an elastic stiffness higher than $10 \%$ of that of the indenter and a yield strain higher than $1 \%$, as well as to penetration depths lower than $\sim 5$ times the characteristic tip defect length of the indenter. We have experimentally tested such a material (an amorphous alloy) by nanoindentation. We show that one must consider the combined effects of indenter tip imperfection and indenter deformability to effectively describe the mechanical response of the test, namely the force-displacement curve. To do so, an identification procedure has been carried out by performing numerical simulations (using Finite Element Analyses) with constitutive equations known to describe well the behaviour of the tested material. A straightforward procedure to take into account these two issues is proposed. It consists first in taking a deformable indenter in the numerical simulations.

V. Keryvin

Univ. Bretagne Sud, FRE CNRS 3744, IRDL, F-56321 Lorient, France

E-mail: vincent.keryvin@univ-ubs.fr

L. Charleux

Univ. Savoie Mont Blanc, EA 4114, SYMME, F-74000 Annecy, France

C. Bernard

Univ. Bretagne Sud, FRE CNRS 3744, IRDL, F-56321 Lorient, France

M. Nivard

Univ. Rennes 1, UMR CNRS 6251, IPR, F-35042 Rennes, France 
It consists also in modifying the experimental curve by taking into account a truncated length to create artificially the material's response to perfectly sharp indentation. This truncated length is determined directly from the loading part of the force-displacement curve. It is also shown that ignoring one or both of these issues results in poor identifi-

5 cation results as well as very different extracted plastic parameters.

Keywords Nanoindentation $\cdot$ Indenter deformability $\cdot$ Tip defect $\cdot$ Hard material $\cdot$ Stiff Material

\section{List of symbols}

E Young's modulus

$v \quad$ Poisson's ratio

$Y_{c} \quad$ Compressive yield strength

$\epsilon_{y}^{c} \quad$ Compressive yield strain

$\varphi \quad$ Friction angle (Drucker-Prager yield criterion)

$P \quad$ Indentation force

$\delta \quad$ Indentation depth

C Indentation loading pre-factor

$\Delta \delta \quad$ Indenter truncated length

$R \quad$ Indenter tip radius

$\beta \quad$ Indenter equivalent complementary angle

$\mathscr{L} \quad$ Residual of the identification procedure

\section{Introduction}

Instrumented indentation (IIT) is a versatile mechanical test that allows one to probe the mechanical behaviour of materials [1]. It is commonly used to extract an indentation elastic modulus from analysing the unloading part of the force-displacement curve [2]. It can also be employed to extract mechanical parameters from constitutive equations aimed at describing the inelastic behaviour of materials (plasticity, viscoplasticity...) 
[3]. IIT can be used at shallow depths (typically below $100 \mathrm{~nm}$ ) with the aid of modern and powerful sensing devices. This nano indentation technique offers the possibility to probe the mechanical behaviour at small depths in fibres, thin films or in bulk brittle materials with a low load to prevent the onset of cracking [1,4]. Many modelings of

5 IIT assume that the indenter (commonly diamond) will not deform during the test since the indenter stiffness is generally much higher than that of the material. This is the assumption made for most reverse analysis methods (see e.g. $[5,6,7,8]$ ). However, this classical assumption is questionable when the material is either very hard (more than 10 GPa in Meyer's hardness) or very stiff (more than 100 GPa in Young's modulus) or even both. This is the first issue examined in this paper.

In some cases, IIT is used at very small depths, for example on hard materials or coatings. This raises an additional issue. It deals with the blunted tip of the indenter. This tip defect can be neglected on IIT results for large enough depths of penetration [9] but this can be questionable at shallower depths. This issue is even more important when the tip is used repeatedly so that the size of its defect [9] is increased. Anyway, at these small depths (with respect to the indenter bluntness), the material is not indented by a sharp indenter but rather by a blunt one. In such cases, the geometrical similarity that results in having a parabola for the loading part of the force-displacement curve is lost. In other words, we have a length scale introduced by the indenter. Various methods [10, $11,12,13]$ have been developped in the past to take into account the tip defect when it comes to estimating the material's stiffness by analysing the unloading part of the indentation curve. However, these methods are not transposable for the determination of plastic parameters from constitutive equations. Moreover, some numerical studies [14] demonstrate the strong influence of the tip defect for shallow penetration depths. hard material. We will propose a way to circumvent the second issue and we will show that the deformability of the indenter must be also taken into account. The originality of this paper is to address simultaneously these two issues and to use a dedicated procedure for extracting plastic parameters by IIT on a real material. 


\section{Experimental and numerical methods}

2.1 Material and indentation procedures

An iron-based amorphous alloy (or bulk metallic glass) with a nominal composition of $\mathrm{Fe}_{41} \mathrm{Co}_{7} \mathrm{Cr}_{15} \mathrm{Mo}_{14} \mathrm{C}_{15} \mathrm{~B}_{6} \mathrm{Y}_{2}$ (at.\%) is studied in this paper. This material is chosen for the

5 purposes of this study since it shows adequate features : it is homogeneous, has an isotropic mechanical behaviour, does not show any length scale for the indentation test nor any cracking features, is hard, stiff [15] and can be modeled nicely and easily by classical plasticity models (assuming it behaves like Zr- or Pd-based metallic glasses as in [16]). The glass transition temperature is $838 \mathrm{~K}$ and the crystallisation temperature is $876 \mathrm{~K}$ [17]. The elastic properties of this material have been reported elsewhere [18]. The Young's modulus and Poisson's ratio are respectively $E=226 \pm 15 \mathrm{GPa}$ and $v=$ $0.337 \pm 0.023$. Instrumented indentation tests were carried out with a nano-indenter testing device (TI950, Hysitron, USA) at ambient conditions $\left(23^{\circ} \mathrm{C}\right.$ and $55 \%$ relative humidity). The indenter tip is a modified Berkovich diamond pyramid. Both AFM (Atomic Force Microscopy, Bruker, Nanoscope V, USA) imaging using a procedure found in [19], and a standard indenter tip calibration method on a fused quartz standard sample [10] lead to an indenter tip radius value of $\sim 260 \mathrm{~nm}$.

Nano-indentation tests were carried out on a dedicated sample, with a '10-10-10' loading sequence: $10 \mathrm{~s}$ to reach the maximum load $P_{m}, 10 \mathrm{~s}$ of holding time, and 10 $s$ to unload the sample's surface. They were load-controlled and the $P_{m}$ value was 10 $\mathrm{mN}$. Due to the high reproducibility of the nano-indentation test on the glass surface, five indents were performed. All imprints were free of cracks, since the critical load for cracking is $\sim 5 \mathrm{~N}[18]$.

\subsection{Numerical procedures}

Finite Element simulations of the indentation process were performed using a twodimensional axisymmetric model with a sample and an indenter. The sample is decomposed into a core zone, underneath the indenter tip, where the mesh is fine, and a shell 
zone where the mesh is coarse. The core zone is itself decomposed into a square zone with 32x32 square elements and a outer zone with quadrangle elements (32 again along the axis $z=0$ ). The shell zone is decomposed into a transition zone and a outer zone, both with quadrangle elements.

All elements are linear. The dimensions of the mesh are chosen to minimize the effect of the far-field boundary conditions. This is made by using a sufficient number of outer elements in the shell zone. The typical ratio of the maximum contact radius and the sample size is about $2 \times 10^{3}$. The indenter is modelled as a perfect cone exhibiting an half-angle $\Psi=70.29^{\circ}$ to match the theoretical projected area function of the modified Berkovich indenter. Its mesh is the same as that of the sample with a geometrical transformation accounting for the geometry of the indenter. The indenter material is assumed to be isotropic, linear elastic (Poisson's ratio of 0.07 and Young's modulus of $1100 \mathrm{GPa}$ ). The contact between the indenter and the sample's surface is strict (Signorini conditions) and taken as frictionless. The contact zone will take place along the square elements of the core zone. The boundary conditions consist in a null displacement on the outer nodes of the sample, in addition to the axisymmetry condition along the vertical axis. The force on the indenter, $P$ (taken as positive), is controlled and the displacement of the indenter far from its tip, $\delta$ (counted positively), is recorded. The boundary-value problem is solved using the commercial software ABAQUS ${ }^{\mathrm{TM}}$ (version 6.10). The preand post-processing tasks were made with the toolbox Abapy [20]. Details and views of the meshes are also visible in the documentation associated with Ref. [20].

The constitutive behaviour of this iron-based amorphous alloy is assumed to be described by a linear isotropic elasticity (parameters E - Young's modulus - and $v$ - Poisson's ratio) followed by rate-independent (with a plastic multiplier $\dot{\lambda}$ in Eq. (1)) perfectly (no strain hardening) plastic flow with a threshold described a Drucker-Prager yield criterion ( $f$ in Eq. (1)) in the stress space ( $\sigma$ is the Cauchy stress tensor). The plastic flow rule 
( $\dot{\epsilon}^{p}$ in Eq. (1)) is assumed to be associated i.e. the dilatancy angle is equal to the friction angle $\varphi[21]$, and $\dot{\lambda}$ is the plastic multiplier :

$$
\left\{\begin{array}{l}
f\left(\underset{\sim}{\sigma} ; Y_{c}, \varphi\right)=\sigma_{\mathrm{eq}}^{\mathrm{VM}}-p \tan \varphi-\left(1-\frac{\tan \varphi}{3}\right) Y_{c} \leq 0 \\
{\underset{\sim}{\epsilon}}^{p}=\dot{\lambda} \frac{\partial f}{\partial \underset{\sim}{\sigma}}=\dot{\lambda}\left(\frac{3}{2} \frac{\underset{\sim}{s}}{\sigma_{\mathrm{eq}}^{\mathrm{VM}}}+\frac{1}{3} \operatorname{tr}(\underset{\sim}{\sigma}) \underset{\sim}{i}\right)
\end{array}\right.
$$

where $\underset{\sim}{s}$ is the deviatoric part of $\underset{\sim}{\sigma}, \sigma_{\mathrm{eq}}^{\mathrm{VM}}=\sqrt{\frac{3}{2} \operatorname{tr}(\underset{\sim}{s} \cdot \underset{\sim}{s})}$ the von Mises equivalent shear stress in tension, $\underset{\sim}{i}$ is the second-order unit tensor, $p=-\frac{1}{3} \operatorname{tr}(\underset{\sim}{\sigma})$ the hydrostatic pressure,

5 tr the trace operator, and $Y_{c}$ the compression yield strength.

The use of such a constitutive model for metallic glasses has already been discussed in the literature for instance on Zr- or Pd-base metallic glass[22, 23, 24, 25]. Our aim in this paper is not to show that the Fe-base alloy follows the same behaviour but rather to use a simple and robust constitutive equation able to model nicely the experimental results. Accordingly, the numerical values of the parameters will not be discussed in depth in terms of material's behaviour.

With the aim of obtaining a match between experimental and numerical results (the force-displacement curves), an automated material parameters identification procedure based on a hybrid method (Levenberg-Marquardt, gradient and Newton-Raphson algorithms, SiDoLo software) [3] is used. Imposing the same loading conditions (force versus time) as the experiment, the square of the difference between the model (sim) and the experimental values $\left({ }^{*}\right)$ is evaluated at the $M_{q}$ instants of observation $t_{i}$ on the displacement $\delta$, by the residual $\mathscr{L}$ for a given value of the set of material parameters $A=\left(Y_{c}, \varphi\right)$ :

$$
\mathscr{L}(A)=\frac{1}{M_{q}} \sum_{i=1}^{M_{q}}\left(\delta^{\operatorname{sim}}\left(t_{i}\right)-\delta^{*}\left(t_{i}\right)\right)^{2}
$$

The identification procedure consists in finding the minima in $\mathscr{L}(A)$.

Another way to proceed would have been to fit both the loading and unloading stages. The former with a parabola (one parameter, the loading prefactor $C$ ), the latter by a power-law fit including the maximum load $P_{m}$, the final depth $\delta_{f}$ and an exponent 
$m$ (or with the ratio of reversible and irreversible works to the total work [26]). This choice was not made in this paper.

\section{Results and discussion}

\subsection{Experimental results}

5 The mechanical response of the indentation test is the force $P$ vs. the displacement $\delta$ (counted positively). Figure 1 presents the experimental results on the Fe-base glass. The curves are highly reproducible. A way to qualify the bluntness of the tip, without imaging it by AFM, an issue raised in Section 2.1, is to calculate a truncated length [11]. The truncated tip defect length, $\Delta \delta$, is obtained straightforwardly by plotting $\sqrt{P}$ vs. $\delta$ for the fused quartz reference sample during the loading stage (increasing $P$ ) or for the iron-based amorphous alloy. This curve should be linear with its origin at $(0,0)$ for a perfect tip (similitude regime, self similarity of sharp indentation, see e.g. $[27,26,4]$ ). This is not the case for shallow depths below $\sim 50 \mathrm{~nm}$, so $\Delta \delta$ was calculated by taking the intercept of a linear fit of this curve for high values of $\delta$, as seen in Figure 2. $\Delta \delta$ is found to be $\sim 15 \mathrm{~nm}$. The loading prefactor $C$ (the square of the slope found for depths higher than $50 \mathrm{~nm}$ in Figure 2) is found to be $274 \pm 2 \mathrm{GPa}$.

\subsection{Numerical results}

\subsubsection{Estimation of initial material parameters}

We will use the elastic parameters discussed hereinbefore ( $\mathrm{E}=225 \mathrm{GPa}, v=0.337)$.

The compressive yield strength is estimated taking the quasi-universal compressive yield strain for iron-based alloys, which is $\epsilon_{y} \sim 2 \%$ [29] (for a reduced temperature $\mathrm{T}(=293$ $\mathrm{K}) / \mathrm{T}_{g}=0.35$ ), so that $\mathrm{Y}_{c}=4500 \mathrm{MPa}$. As for the friction coefficient it will estimated at $\sim 10^{\circ}$, which is value commonly found for metallic glasses [30]. 
3.2.2 Creating the material's response for a perfectly sharp tip

To identify the material parameters of the constitutive equation, we will compare the numerical simulation results to the experimental data. The latter will be shifted to higher depths by $\Delta \delta$. Such a method allows one to capture the material's response to sharp IIT

5 had the indenter been perfectly sharp. This comparison holds nevertheless for depths higher than $\sim 2-3$ times the tip defect. To our knowledge, this very method is not used for identifying plastic parameters by numerical simulations. To assess this methodology, we have performed some numerical simulations (displacement controlled). We have used a rigid indenter with a blunted tip modelled as spherical with a radius of $260 \mathrm{~nm}$, in accordance with the experimental AFM measurements and tip calibration described in Section 2.1. A schematics of such a geometry is shown in Figure 3. The spherical part (in red) and the conical part (in blue) are joined so that they have the same slope at point T. The tip defect length (td) is given by the radius of the spherical tip $(R=260 \mathrm{~nm})$ and the angle between the horizontal axis and the conical part $\left(\beta=19.7^{\circ}\right)$. In our case td is $16 \mathrm{~nm}$. The vertical distance between the horizontal axis and point $\mathrm{T}\left(\mathrm{z}_{t}\right)$ is $15 \mathrm{~nm}$.

The material is elasto-plastic $\left(\mathrm{J}_{2}\right.$ plasticity without strain hardening with the parameters described hereinbefore save the friction coefficient $\varphi$ taken as null) and the maximum penetration depth is $292 \mathrm{~nm}$. The fit of the loading stage of the resulting loaddisplacement gives a truncated length $\Delta \delta$ of $\sim 15 \mathrm{~nm}$ in accordance with the tip defect length $\mathrm{td}=16 \mathrm{~nm}$, as well as AFM measurements of this defect length (15 $\mathrm{nm}$ as well). Then, another simulation is run but with a perfectly sharp indenter with a maximum penetration depth increased by $15 \mathrm{~nm}$ i.e. $307 \mathrm{~nm}$. The results of these two simulations are presented in Figure 4 along with those of the first one shifted by $15 \mathrm{~nm}$ to the right. It is obvious from the comparison between the sharp indentation and the shifted blunted one that these two curves are the same for depths higher than 2-3 times the truncated length $\Delta \delta$. It validates numerically the way to create the material's response to perfectly sharp indentation. 


\subsubsection{Taking into account the indenter tip defect and its deformability}

The experimental results (shifted by $\Delta \delta$ ) are shown along with a numerical simulation with identified parameters (reported in Table 1, (d)). A very close match between these two curves is reported, which is shown in Figure 5. The very small value of the residual

5 (0.6) is also indicative of the quality of the match. The identified parameters are in accordance with literature results.

With the same material parameters, we have ran a simulation by considering this time the indenter to be rigid. The force-displacement curve is plotted on the same Figure 5 exhibiting a stiffer response as expected. The loading pre-factor, $C$, which was $274 \mathrm{GPa}$ for the simulation with the deformable indenter is now $301 \mathrm{GPa}$, with the rigid one, that is $10 \%$ higher.

We have then carried out three other identification procedures:

- a first one, referred to as (a), without taking into account the tip defect (therefore without shifting the experimental data by the truncated length $\Delta \delta$ ) and with a rigid indenter,

- a second one, referred to as (b), without taking into account the tip but with a deformable indenter,

- a third one, referred to as (c), by taking into account the tip (therefore by shifting the experimental data by the truncated length $\Delta \delta$ ) and with a rigid indenter.

The results of these identifications are shown in terms of material parameters and residuals in Table 1, as well as in Figure 6. No identification (from these three) was able to match the experiments. Not taking into account the tip defect, cases (a) and (b), is the main origin of these discrepancies. This situation would be even more exacerbated for IIT tests at shallower penetration depths. Moreover, case (c) shows that taking into account the tip defect but not the indenter deformability is also not enough.

Taking into account the tip defect for the analysis of the whole force-displacement curve can be explicitly made $[9,31]$. It consists in imaging the real geometry of the indenter, export it to a computer-aided design software and/or to mesh it before running a Finite-Element analysis. This procedure, however straightforward it might be, is 
somehow tedious and time-consuming since it requires fine AFM imaging and a threedimensional numerical simulation. In contrast, the method proposed in this work is effortless and requires only a straightforward fit of the loading step of indentation.

As for the indenter deformability, one has to use a deformable indenter in the nu5 merical simulations. The identification results of case (c) show that it is mandatory.

\section{Concluding remarks}

We have performed instrumented nano-indentation tests on an amorphous alloy (or metallic glass) of the FeC family. This material has been chosen since it is both very stiff (as steel) and very hard (as single crystal quartz), it is isotropic, homogeneous, exhibiting no length scale for the range of penetration depths studied, and also because its mechanical behaviour is known to be adequately described by simple constitutive equations involving a small number (2) of plastic properties. We have carried out an identification procedure for extracting the plastic properties by minimising the error between the experimental force-displacement curve and the numerical one. The former has been modified to take into account the truncation of the indenter tip by simply shifting to higher penetration depths the raw data by an amount called truncated length, which is determined straightforwardly by fitting the loading stage of the force-displacement curve. The latter has been obtained by performing Finite Element Analyses with a deformable indenter. The identification procedure allowed one to perfectly match the experimental data and the identified parameters are very consistent to what is expected from the literature data. It has moreover been validated numerically.

It is also shown that the identification procedure fails when one does not take into account the tip defect or the indenter deformability or both. The present results obtained on a stiff and hard material clearly shows the necessity to consider the tip defect of the indenter as well as its deformability, when it comes to propose a constitutive model able to describe quantitatively the mechanical response of the indentation test. The results obtained with this particular material can be generalised. The higher the yield strain and the elastic stifness, the more mandatory it is to consider the indenter as deformable. 
Acknowledgements We acknowledge financial support from the program CPER PRIN2TAN for the Hysitron nanoindentation apparatus. We would like to thank Prof. Jun Shen (Harbin Institute of Technology, China) for providing the samples, Dr. J.-P. Guin (CNRS, France) for the AFM measurements and Prof. P. Pilvin for advice on the identification procedure.

\section{References}

1. A. C. Fischer-Cripps, Introduction to Contact MEchanics. Mechanical Engineering Series, Berlin, Heidelberg: Springer Berlin Heidelberg, 2006. 2, 3

2. W. Oliver and G. Pharr, "An improved technique for determining hardness and elastic modulus using load and displacement sensing indentation experiments," J. Mater. Res., vol. 7, pp. 1564-1583, jan 1992. 2

3. A. Andrade-Campos, S. Thuillier, P. Pilvin, and F. Teixeira-Dias, "On the determination of material parameters for internal variable thermoelastic-viscoplastic constitutive models," Int. J. Plast., vol. 23, no. 8 , pp. 1349-1379, 2007. 3, 6

4. A. C. Fischer-Cripps, Nanoindentation. Berlin, Heidelberg: Springer, 2011. 3, 7

5. A. Giannakopoulos and S. Suresh, "Determination of elastoplastic properties by instrumented sharp indentation," Scr. Mater., vol. 40, pp. 1191-1198, apr 1999. 3

6. M. Dao, N. Chollacoop, K. J. Van Vliet, T. A. Venkatesh, and S. Suresh, "Computational modeling of the forward and reverse problems in instrumented sharp indentation," Acta Mater., vol. 49, no. 19, pp. 3899-3918, 2001. 3

7. O. Casals and J. Alcalá, "The duality in mechanical property extractions from Vickers and Berkovich instrumented indentation experiments," Acta Mater, vol. 53, pp. 3545-3561, nov 2005. 3

8. J. Lee, C. Lee, and B. Kim, "Reverse analysis of nano-indentation using different representative strains and residual indentation profiles," Mater. Des., vol. 30, pp. 3395-3404, oct 2009. 3

9. A. W. Warren and Y. B. Guo, "Machined surface properties determined by nanoindentation: Experimental and FEA studies on the effects of surface integrity and tip geometry," Surf. Coatings Technol., vol. 201, no. 1-2, pp. 423-433, 2006. 3, 9

10. W. Oliver and G. Pharr, "Measurement of hardness and elastic modulus by instrumented indentation: Advances in understanding and refinements to methodology," J. Mater. Res., vol. 19, pp. 3-20, mar 2004. 3,4

11. G. Hochstetter, A. Jimenez, and J. Loubet, "Strain-rate effects on hardness of glassy polymers in the nanoscale range. Comparison between quasi-static and continuous stiffness measurements," $J$. Macromol. Sci. part B, vol. 38, no. 5-6, pp. 681-692, 2006. 3, 7

12. B. Poon, D. Rittel, and G. Ravichandran, "An analysis of nanoindentation in elasto-plastic solids," Int. J. Solids Struct., vol. 45, pp. 6399-6415, dec 2008. 3 
13. B. Poon, D. Rittel, and G. Ravichandran, "An analysis of nanoindentation in linearly elastic solids," Int. J. Solids Struct., vol. 45, no. 24, pp. 6018-6033, 2008. 3

14. T. H. Wang, T. H. Fang, and Y. C. Lin, "A numerical study of factors affecting the characterization of nanoindentation on silicon," Mater. Sci. Eng. A, vol. 447, no. 1-2, pp. 244-253, 2007.3

5 15. V. Keryvin, X. Vu, V. Hoang, and J. Shen, "On the deformation morphology of bulk metallic glasses underneath a Vickers indentation," J. Alloys Compd., vol. 504, pp. S41-S44, aug 2010. 4

16. V. Keryvin, "Indentation as a probe for pressure sensitivity of metallic glasses.," J. Phys. Condens. Matter, vol. 20, p. 114119, mar 2008. 4

17. J. Shen, Q. Chen, J. Sun, H. Fan, and G. Wang, "Exceptionally high glass-forming ability of an FeCoCrMoCBY alloy," Appl. Phys. Lett., vol. 86, no. 15, p. 151907, 2005. 4

18. V. Keryvin, V. H. Hoang, and J. Shen, "Hardness, toughness, brittleness and cracking systems of an iron-based bulk metallic glass by indentation," Intermetallics, vol. 17, no. 4, pp. 211-217, 2009. 4

19. M. R. VanLandingham, T. F. Juliano, and M. J. Hagon, "Measuring tip shape for instrumented indentation using atomic force microscopy," Meas. Sci. Technol., vol. 16, no. 11, pp. 2173-2185, 2005.

20. L. Charleux, V. Keryvin, and L. Bizet, "abapy: Abapy_v1.0," 2015. 5

21. W.-F. Chen and D.-J. Han, Plasticity for Structural Engineers. Florida, USA: J. Ross Publishing Classics, 2007. 6

22. P. E. Donovan, "Plastic flow and fracture of Pd40Ni40P20 metallic glass under an indentor," J. Mater. Sci., vol. 24, pp. 523-535, feb 1989. 6

23. M. N. M. Patnaik, R. Narasimhan, and U. Ramamurty, "Spherical indentation response of metallic glasses," Acta Mater., vol. 52, no. 11, pp. 3335-3345, 2004. 6

24. V. Keryvin, R. Crosnier, R. Laniel, V. H. Hoang, and J.-C. Sangleboeuf, "Indentation and scratching mechanisms of a ZrCuAlNi bulk metallic glass,” J. Phys. D. Appl. Phys., vol. 41, p. 074029, apr 2008.

25. J. Brest, V. Keryvin, P. Longère, and Y. Yokoyama, "Insight into plasticity mechanisms in metallic glasses by means of a Brazilian test and numerical simulation," J. Alloys Compd., vol. 586, pp. S236S241, feb 2014. 6

26. Y. T. Cheng and C. M. Cheng, "Scaling, dimensional analysis, and indentation measurements," Mater. Sci. Eng. R Reports, vol. 44, no. 4-5, pp. 91-150, 2004. 7

27. D. Tabor, "The physical meaning of indentation and scratch hardness," Br. J. Appl. Phys., vol. 7, no. 5, p. $159,1956.7$

28. L. Charleux, V. Keryvin, M. Nivard, J.-P. Guin, J.-C. Sangleboeuf, and Y. Yokoyama, "A method for measuring the contact area in instrumented indentation testing by tip scanning probe microscopy imaging," Acta Mater., vol. 70, pp. 249-258, may 2014.

29. R. T. Qu, Z. Q. Liu, R. F. Wang, and Z. F. Zhang, "Yield strength and yield strain of metallic glasses and their correlations with glass transition temperature," J. Alloys Compd., vol. 637, pp. 44-54, 2015. 7 
30. V. Keryvin, K. Eswar Prasad, Y. Gueguen, J.-C. Sanglebœuf, and U. Ramamurty, “Temperature dependence of mechanical properties and pressure sensitivity in metallic glasses below glass transition," Philos. Mag., vol. 88, pp. 1773-1790, apr 2008. 7

31. K. Gadelrab, F. Bonilla, and M. Chiesa, "Densification modeling of fused silica under nanoindentation," J. Non. Cryst. Solids, vol. 358, pp. 392-398, jan 2012. 9 


\begin{tabular}{lcccc}
\hline \hline & $Y_{c}[\mathrm{MPa}]$ & $\epsilon_{y}^{c}[\%]$ & $\varphi\left[^{\circ}\right]$ & Residual $\mathscr{L}$ (a.u.) \\
\hline (a) & 5200 & 2.31 & 20 & 15 \\
(b) & 1835 & 0.816 & 41 & 26 \\
(c) & 5120 & 2.28 & 8 & 13 \\
(d) & 3690 & 1.64 & 22 & 0.6 \\
\hline \hline
\end{tabular}

Table 1. Results of the four different identification procedures in terms of material parameters. $Y_{c}$ (compressive strength) and $\varphi$ (friction angle) are the identified plastic parameters (see Eq. (1)); $\epsilon_{y}^{c}=Y_{c} / E$ is the compressive yield strain; $\mathscr{L}$ is the residual of the identification procedure (see Eq. (2)). Case (a) is when taking into account neither the indenter tip defect nor its deformability. Case (b) is when taking into account only the indenter deformability. Case (c) is when taking into account only the tip defect. Case (d) is when taking into account the indenter tip defect and its deformability. Note that both E, Young's modulus, and v, Poisson's ratio were kept constant at $225 \mathrm{GPa}$ and 0.337 , respectively. 
Instrumented indentation tests at very low depths on stiff and hard materials

15

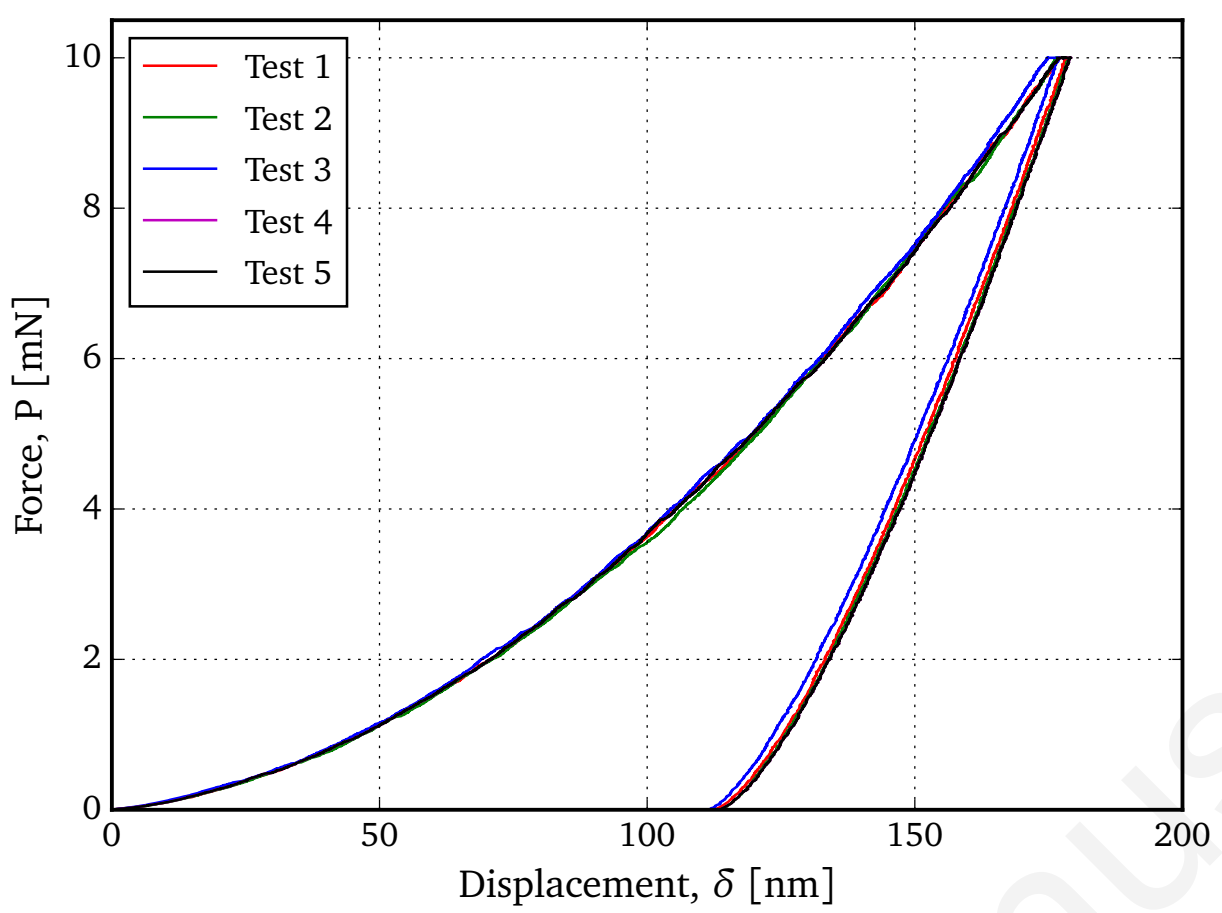

Fig. 1. Force-displacement curves of the Fe-base metallic glass under a nanoindentation test (five tests). 


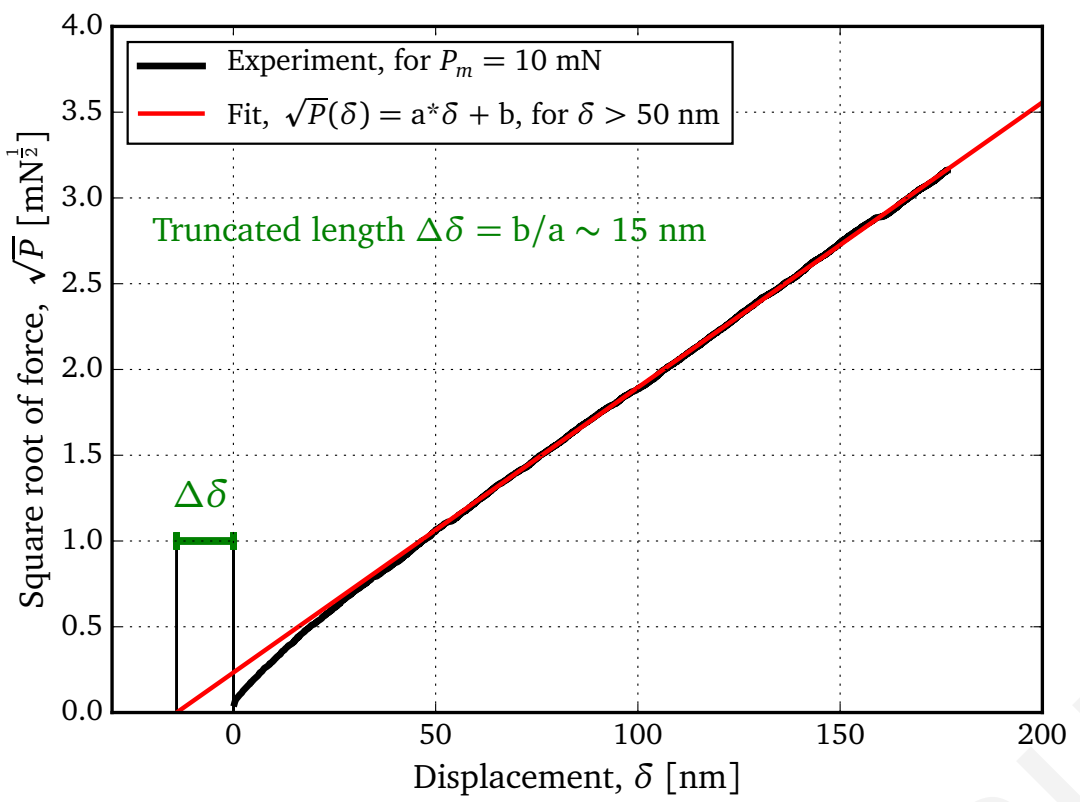

Fig. 2. Evolution of the square root of the force versus the indentation depth during the loading stage of a $10 \mathrm{mN}$ indentation test on the Fe-base glass. A linear fit for depths higher than $50 \mathrm{~nm}$ (for which we are in the similitude regime) is extrapolated down to the $x$-axis to give the tip defect in terms of a truncated length $\Delta \delta$ $\sim 15 \mathrm{~nm}$. 


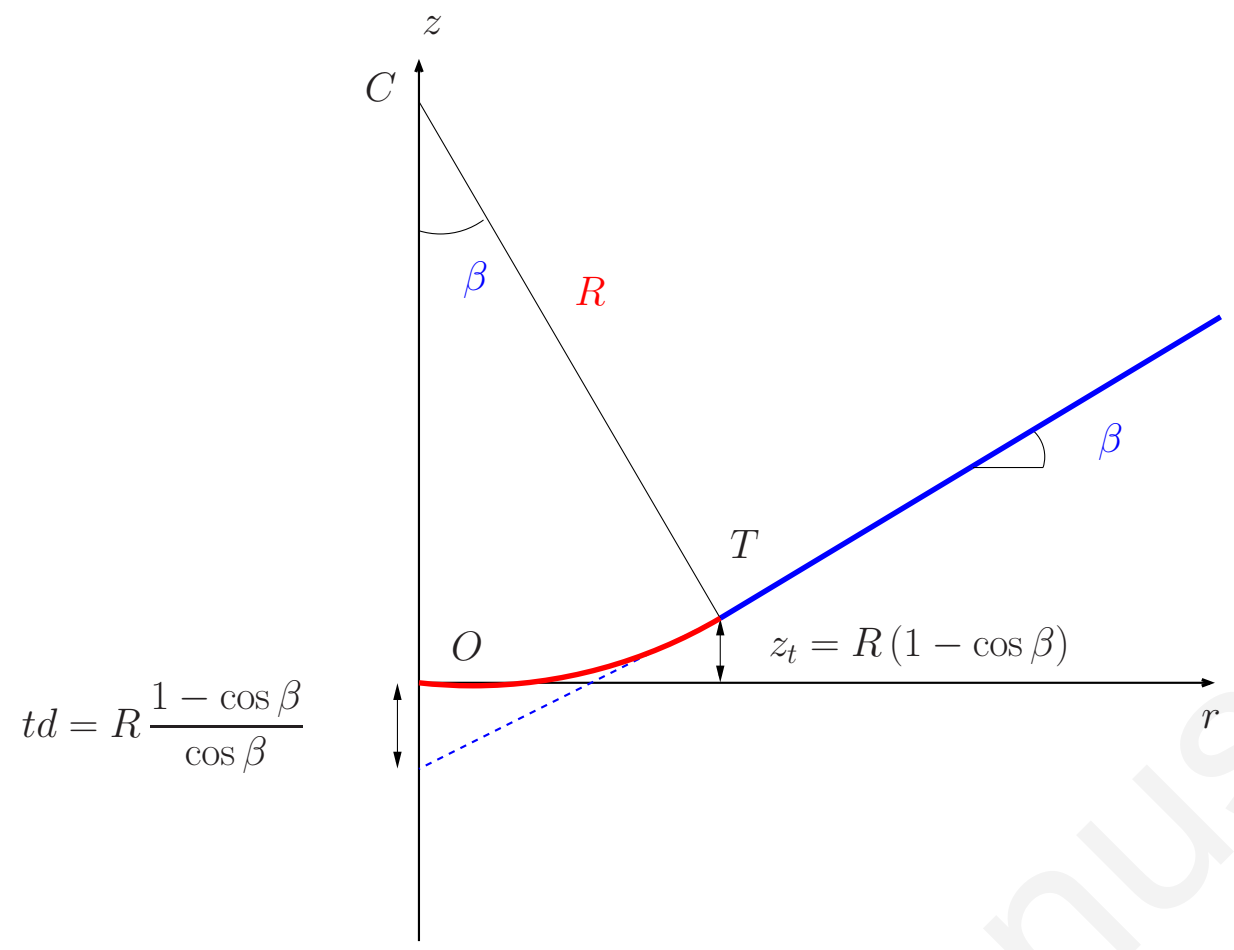

Fig. 3. Schematic of a blunted indenter modelled as sphero-conical. The spherical part (in red) and the conical part (in blue) are joined so that they have the same slope at point $T$. The tip defect length ( $t d$ ) is given by the radius of the spherical tip $(R)$ and the angle between the horizontal axis and the conical part $(\beta)$. The vertical distance between the horizontal axis and point $T$ is $z_{t}$ 


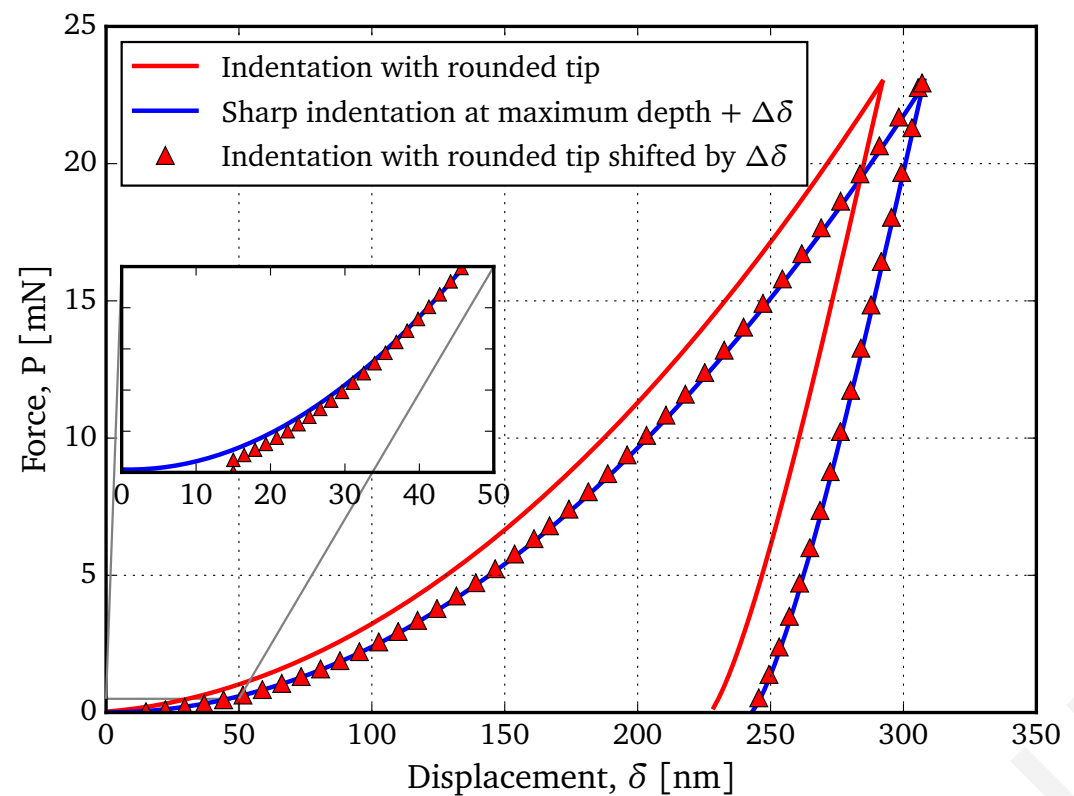

Fig. 4. Numerical simulations (force-displacement curves) with a rounded tip of $260 \mathrm{~nm}$ (in accordance with AFM measurements) at a given arbitrary penetration depth $\delta_{m}=292 \mathrm{~nm}$ and a perfectly sharp tip (at a penetration depth $\delta_{m}+\Delta \delta$, where $\Delta \delta$ is the truncated length (found by fitting the loading stage of the rounded tip simulation, here $15 \mathrm{~nm}$ ). The results from the rounded tip simulation, shifted by $\Delta \delta$ to higher penetration depths, overlap those with the perfect tip. The inset indicates furthermore that this is only valid for $\delta$ greater than $\sim 40 \mathrm{~nm}$ that is $\sim 2-3$ times $\Delta \delta$. 


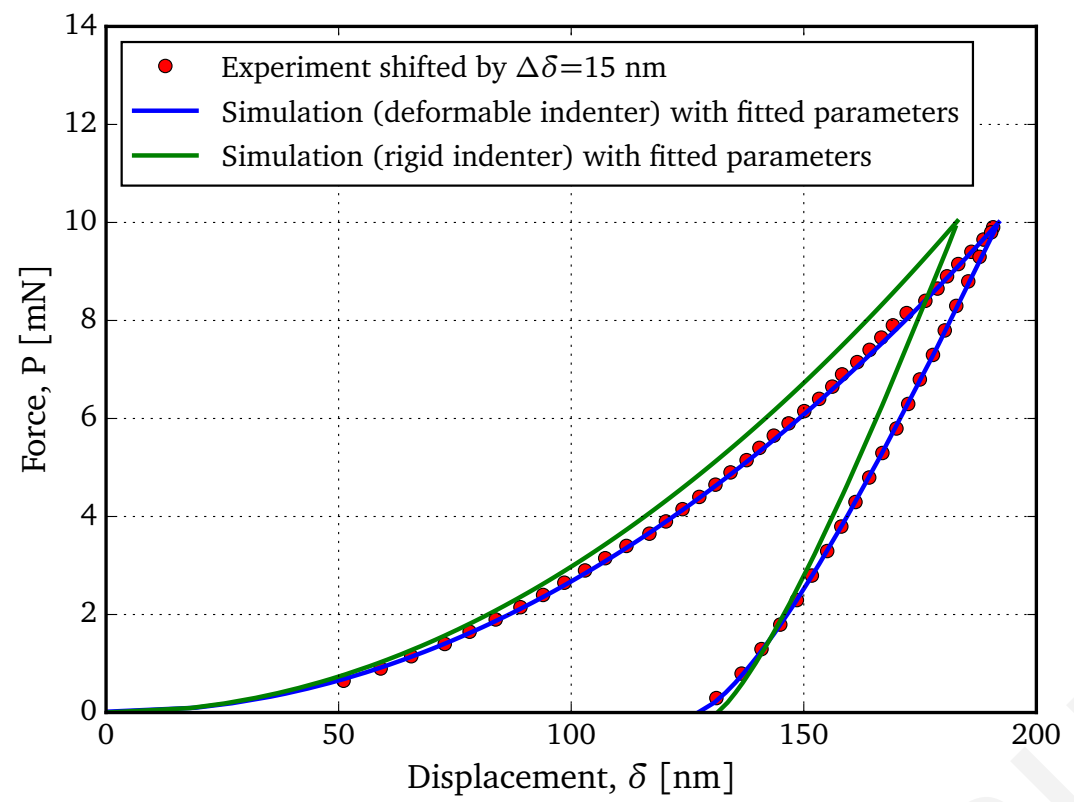

Fig. 5. Results of the identification procedure (d). The force-displacement curve obtained from numerical simulations with the parameters found in Table 1 (d), taking into account the indenter deformability matches the experimental curve (shifted by the truncated length $\Delta \delta=15 \mathrm{~nm}$ ). The results of a direct simulation with the same material parameters but with a rigid indenter are also shown to highlight the dramatic influence if the indenter deformability for hard and stiff materials. 


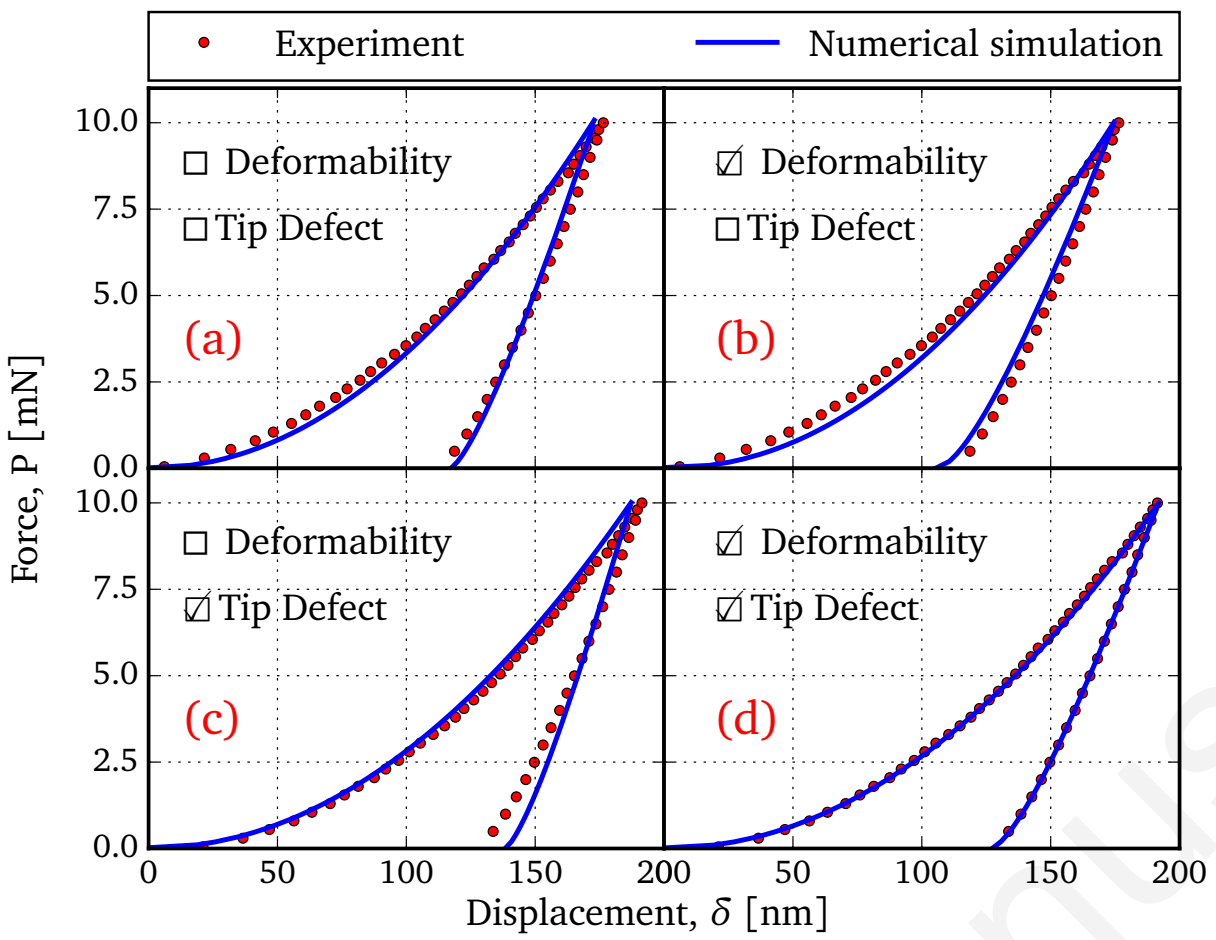

Fig. 6. Results of the four different identification procedures in terms of force-displacement curves (experiment versus numerical simulation). Case (a) is when taking into account neither the indenter tip defect nor its deformability. Case (b) is when taking into account only the indenter deformability. Case (c) is when taking into account only the tip defect. Case (d) is when taking into account the indenter tip defect and its deformability. 